\title{
Phagocytic Activation of Macrophages with Serum MAF Depends on Engulfment Efficiency and Not Migratory Activity
}

\author{
RIHO MASHIBA ${ }^{1}$, MAMI ISHIKAWA ${ }^{1}$, YU SUMIYA ${ }^{1,2}$, KUMPEI KAWAKATSU $^{1}$, \\ NGOC KIET TRAN ${ }^{1}$ and TAKAHITO NISHIKATA ${ }^{1}$ \\ ${ }^{1}$ Frontiers of Innovative Research in Science and Technology (FIRST), Konan University, Kobe, Japan; \\ ${ }^{2}$ Club Cosmetics Co. Ltd, Osaka, Japan
}

\begin{abstract}
Background/Aim: Serum-derived macrophage activating factor (serum MAF) is known to increase the phagocytic activity of macrophages and potentially plays a role in activating cancer immunity. In order to reveal the contributing factors for phagocytic activation, the migratory activity and the efficiency of engulfment was analyzed. Materials and Methods: THP-1 macrophages were induced by 12-O-tetradecanoyl-13-acetate (TPA). The migratory activity and efficiency of engulfment were analyzed by time-lapse imaging and suspension assay, respectively. Results: While the distance of migration did not change before and after activation with serum MAF, the efficiency of beads internalisation was significantly increased. Conclusion: Phagocytic activation of serum-MAF-treated macrophages was caused by increasing the efficiency of engulfment. This study contributes to the knowledge about the activation of the immune system through phagocytic activation of macrophages.
\end{abstract}

Macrophages are key players in primary defense against invading pathogens (1). Macrophages play an important role in the interplay between innate and adaptive immunity as well. They fulfil this role by engulfing pathogens such as bacteria and viruses, presenting antigens, and releasing cytokines (1-3). Thus, the activation of macrophages could activate the entire immune system.

We established a novel experimental system for evaluating the phagocytic activity of THP-1 derived macrophages

This article is freely accessible online.

Correspondence to: Takahito Nishikata, Frontiers of Innovative Research in Science and Technology (FIRST), Konan University, 71-20, Minatojima-minamimachi, Chuo-ku, Kobe 605-0047, Japan. Tel: +81 783031349, Fax: +81 783031495, e-mail: nisikata@konan-u.ac.jp

Key Words: THP-1, serum MAF (serum-derived macrophage activating factor), $\mathrm{pH}$-sensitive chromophore, suspension assay, time-lapse image analysis. quantitatively and efficiently (4). Using this experimental system, the phagocytic activity of macrophages within $60 \mathrm{~min}$ of macrophage activating factor (MAF) treatment can be evaluated. Screening MAFs using this system revealed that the serum MAF (serum-derived macrophage activating factor) was a prominent activator of rapid phagocytic activity (4). Serum MAF is the human serum treated with galactosidase and sialidase (5) and is suspected to have a role in activating cancer immunity (6). Despite extensive clinical studies, the mechanism of activation of macrophages phagocytic activity by serum MAF is poorly understood. In order to investigate the molecular mechanism of phagocytic activation with serum MAF, the physiological changes in macrophages including migratory activity and efficiency of engulfment were examined.

\section{Materials and Methods}

Cells and cell culture. The THP-1 cell lines were obtained from the RIKEN BRC through the National Bio-Resource Project of MEXT (Tokyo, Japan). THP-1 cells were grown in RPMI-1640 medium supplemented with $10 \%$ foetal bovine serum (Gibco, E.U.-approved, South American Origin), 3\% L-glutamine, and 10\% sodium hydrogen carbonate. Cells were cultured in $5 \% \mathrm{CO}_{2}$ and $95 \%$ air in a fully humidified atmosphere at $37^{\circ} \mathrm{C}$. To induce differentiation into macrophage-like cells, THP-1 cells were seeded at a concentration of $2.0 \times 10^{4}$ cells/well in a $96-$ well plate, and $5.0 \times 10^{5}$ cells $/ 35-\mathrm{mm}$ dish and incubated with $200 \mathrm{ng} / \mathrm{mL} \mathrm{12-O-tetradecanoyl-13-acetate}$ (TPA; Sigma-Aldrich, St. Louis, MO, USA) for $24 \mathrm{~h}$.

MAF (macrophage activating factor) reagents. Human recombinant interferon (IFN)- $\gamma$ was obtained from Wako (Osaka, Japan). Lipopolysaccharides (LPS) from Escherichia coli 0111:B4 were purchased from Sigma-Aldrich.

pH-sensitive beads. $\mathrm{pH}$-sensitive beads consisted of Dynabeads ${ }^{\circledR}$, Protein G, (Invitrogen, Oslo, Norway), and pH-sensitive fluorescent probes (AcidiFluor ${ }^{\mathrm{TM}}$ ORANGE-NHS; Goryo chemical Inc.).

Phagocytic assay. The medium of differentiated macrophages in 96well plates was changed to RPMI1640 medium containing MAF (1 $\mu \mathrm{g} / \mathrm{ml} \mathrm{LPS}, 1 \mathrm{ng} / \mathrm{ml} \mathrm{IFN}-\gamma, 8 \mu \mathrm{g} / \mathrm{ml}$ serum MAF) and cultured for $60 \mathrm{~min}$. After pre-activation with MAFs, cells were washed with serum-free RPMI1640, and the medium was changed to $100 \mu \mathrm{l} /$ well of serum-free RPMI 1640 containing $3 \mu \mathrm{g} / \mathrm{well} \mathrm{pH}$-sensitive beads. 
After a 60-min period of phagocytosis, the internalised beads were quantitated by a high-throughput quantitative assay system (7). Phagocytic activities of macrophages were evaluated as per the internalised bead ratio, as measured by fluorescence (IBRf), which was calculated by the following formula (7):

IBRf $(\%)=($ Fluorescent intensity of internalised beads $) /($ Fluorescent intensity of all beads) $\times 100$.

Time-lapse image analyses. Differentiated macrophages in a 35-mm dish were pre-activated with RPMI1640 medium containing MAFs for $60 \mathrm{~min}$. The medium was changed to $2 \mathrm{~mL}$ of serum-free RPMI1640 containing $60 \mu \mathrm{g}$ magnetic beads (Dynabeads ${ }^{\circledR}$ Protein G; Invitrogen, Oslo, Norway). The images of engulfing macrophages were photographed every $4 \mathrm{~min}$ and assembled into a time-lapse sequence. The migratory activity of individual macrophages was quantitated by tracing 6 photos every 12 min using Image-J software (US National Institutes of Health, Bethesda, MD, USA). Three independent experiments were carried out, and three random areas were filmed in each experiment. The efficiency of engulfment was evaluated by the following formula:

Efficiency of engulfment $(\%)=($ number of internalised beads $) /$ (number of beads attached to macrophages) $\times 100$.

The number of attached beads was counted manually using whole time-lapse sequences.

Phagocytic assay in suspension condition. The differentiated and preactivated macrophages were dissociated with Accutase (Innovative Cell Technologies, San Diego, CA, USA) for 10 min. Macrophages were transferred to $1.5 \mathrm{ml}$ tubes with $1 \mathrm{ml}$ of serum-free RPMI 1640 containing $30 \mu \mathrm{g} \mathrm{pH}$-sensitive beads and stirred for $60 \mathrm{~min}$ at $38 \mathrm{rpm}$. The efficiency of engulfment by macrophages was evaluated with IBRf using a high-throughput quantitative assay system (7).

Statistical analyses. Differences between data was determined using a student's $t$-test. Significance was set at $p>0.01$.

\section{Results}

The migratory activity of macrophages was not increased by MAF activation. First, MAFs' induction of phagocytic activity was confirmed using the newly developed assay system (Figure 1) (7). Previous results were reproduced (4, 7). After 60 -min treatment with MAFs (LPS + IFN- $\gamma$ and serum MAF), although both MAFs could activate bead engulfment, activation of phagocytic activity was evident.

The migration distance of macrophages after MAF activation. After $60 \mathrm{~min}$ of MAF (LPS + IFN- $\gamma$ and serum MAF) treatment, the migration distance did not significantly differ from the control, not-treated macrophages (control; $11.1 \pm 0.6 \mu \mathrm{m}$, LPS $+\mathrm{IFN}-\gamma ; 11.6 \pm 0.6 \mu \mathrm{m}$, serum MAF; $12.9 \pm 0.3 \mu \mathrm{m}$ : Figure $2 \mathrm{~A})$. In addition to the migration distance, the number of internalised beads in each cell was counted and the relationship between the distance and the number of internalised beads was plotted (Figure 2B-D). Regardless of the MAF, there was no correlation between the

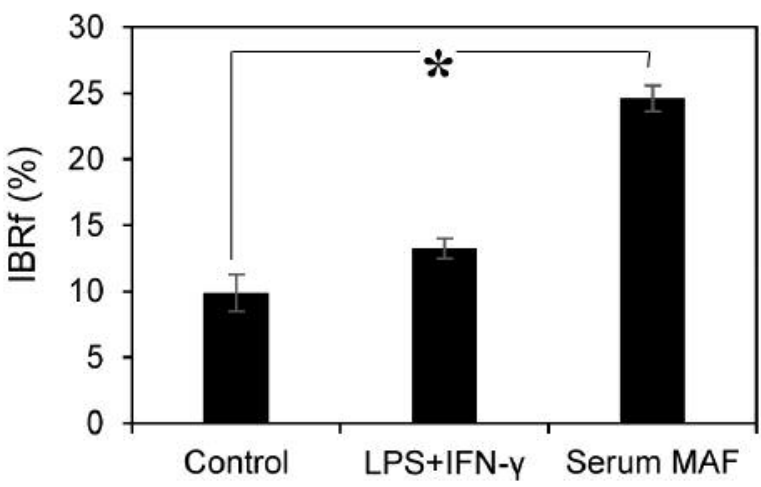

Figure 1. Difference in phagocytic activity of THP-1 macrophages treated with MAFs. Phagocytic activity was evaluated by using fluorescence (IBRf) to determine the internalised bead ratio. Details of these calculations are described in the Materials and Methods section. MAFs used in this experiment are as follows: $1 \mu \mathrm{g} / \mathrm{ml} L P S+1 \mathrm{ng} / \mathrm{ml} I F N-\gamma, 8$ $\mu \mathrm{g} / \mathrm{ml}$ serum MAF. Error bars represent SDs $(n=3)$. * $p=0.0003$ (t-test).

distance travelled and the number of internalised beads. These results suggest that there is no correlation between phagocytic and migratory activity.

The efficiency of engulfment was increased by serum MAF. Next, the number of beads attached to and internalised by macrophages during the 60-min time-lapse recordings was counted. Subsequently the efficiency of engulfment was calculated. When macrophages were activated with LPS + IFN- $\gamma$, all the indices (the number of attached beads, number of internalised beads, and efficiency of engulfment) tended to increase insignificantly, and its activation ratio compared to the control was calculated to be 1.5 (Table I). However, activation with serum MAF significantly increased all indices. Notably, the efficiency of engulfment significantly increased compared to control, and its activation ratio was 2.3. This result clearly demonstrated that serum MAF activated the phagocytic activity of macrophages by increasing the efficiency of bead internalisation.

Therefore, the phagocytic activity was examined in suspension conditions. In suspension conditions, macrophages and beads are continuously stirred, and all macrophages have an equal chance to interact with beads. When macrophages were activated with LPS+IFN- $\gamma$, the amount of internalised beads was almost the same as that of the control $(2.5 \pm 0.2)$ (Figure 3). Conversely, when macrophages were activated with serum MAF, bead internalisation was drastically increased by 6 -fold $(14.7 \pm 1.6)$ over the control.

All these results were obtained by macrophages preactivated with MAFs for $60 \mathrm{~min}$ before the evaluation of phagocytic activity. When macrophages were pre-activated for $4 \mathrm{~h}$ (data not shown), the results were nearly identical to the 60 -min pre-activation. 
A

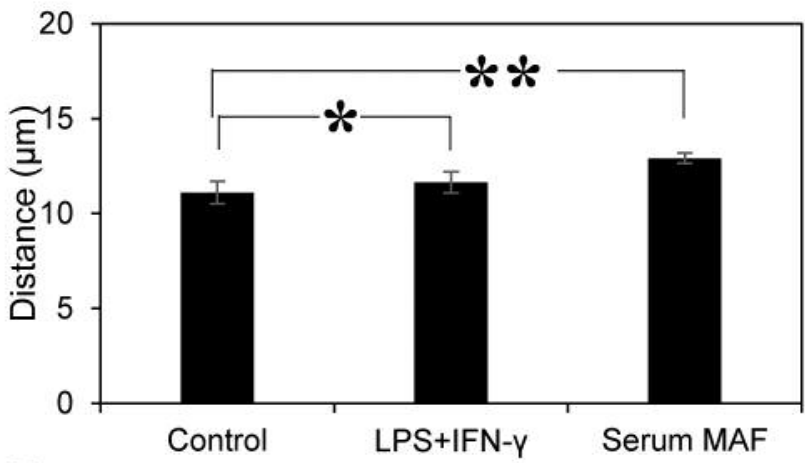

C

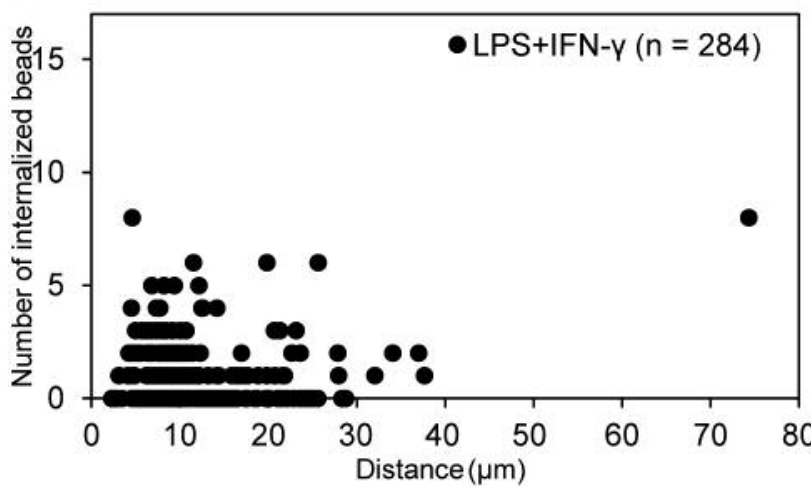

B

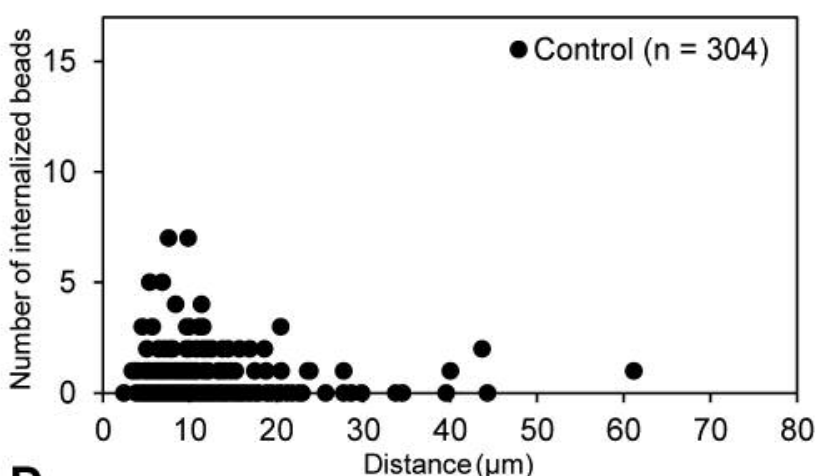

D

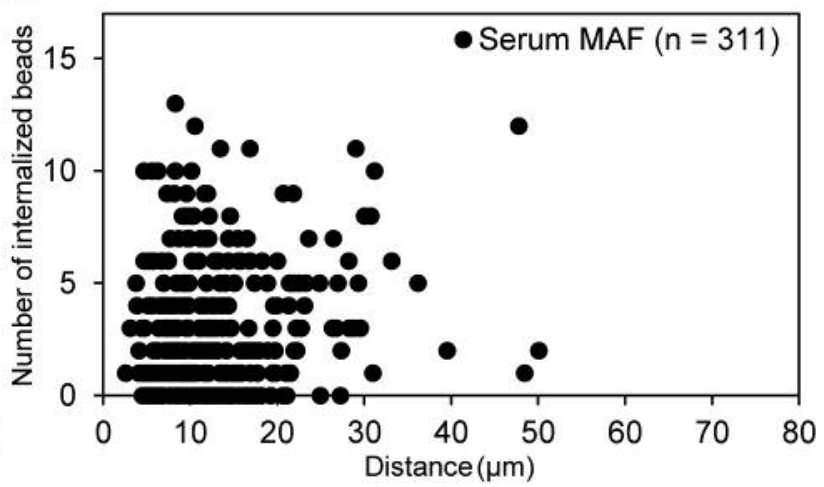

Figure 2. Migratory activity of THP-1 macrophages treated with MAFs. The average migration distance of macrophages was analyzed (A). Error bars represent $S D(n=3) . * p=0.40$, and ${ }^{* *} p=0.02$ ( $t$-test). Plots of migration distance versus the number of engulfed beads of each macrophage are shown (B-D). MAFs used in this experiment are as follows: no MAFs $(B), 1 \mu \mathrm{g} / \mathrm{ml} L P S+1 \mathrm{ng} / \mathrm{ml} I F N-\gamma(C), 8 \mu \mathrm{g} / \mathrm{ml} \operatorname{serum~MAF}(D)$.

Table I. Difference in the efficiency of engulfment of THP-1 macrophages activated using MAFs.

\begin{tabular}{lcccc}
\hline MAFs & $\begin{array}{c}\text { Number of } \\
\text { attached } \\
\text { beads }\end{array}$ & $\begin{array}{c}\text { Number of } \\
\text { internalised } \\
\text { beads }\end{array}$ & $\begin{array}{c}\text { Efficiency } \\
\text { of } \\
\text { engulfment }\end{array}$ & $\begin{array}{c}\text { Activation ratio } \\
\text { of efficiency }(v s . \\
\text { without MAF) }\end{array}$ \\
\hline Without MAF & $1.7 \pm 0.4$ & $0.7 \pm 0.2$ & $37.3 \pm 5.8$ & - \\
LPS + IFN- $\gamma$ & $1.9 \pm 0.3$ & $1.1 \pm 0.3$ & $55.9 \pm 9.9$ & 1.5 \\
Serum MAF & $3.3 \pm 0.5$ & $2.9 \pm 0.5$ & $87.2 \pm 5.7 *$ & 2.3 \\
\hline
\end{tabular}

IDescribed as mean $\pm \mathrm{SD}(\mathrm{n}=3) .{ }^{*} p<0.01$ ( $t$-test $v s$. without MAF).

\section{Discussion}

In our in vitro system, two hypotheses can be proposed to explain the phagocytic activation of macrophages: (1) the migratory activity of macrophages is enhanced and thus macrophages can interact with more beads, or (2) the efficiency of internalisation is enhanced; thus, even with the

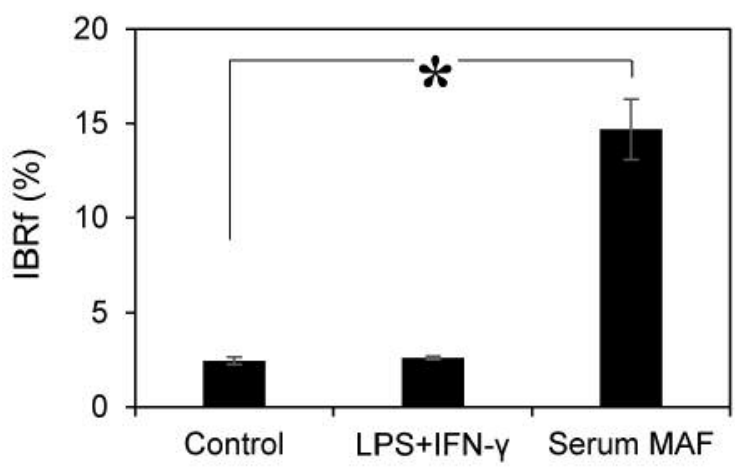

Figure 3. Phagocytic assay treated with MAFs in suspension conditions. $M A F s$ used in this experiment are as follows: $1 \mu \mathrm{g} / \mathrm{ml} \mathrm{LPS}+1 \mathrm{ng} / \mathrm{ml} I F N-$ $\gamma, 8 \mu \mathrm{g} / \mathrm{ml}$ serum MAF. Error bars represent $S D(n=3)$. ${ }^{*} p=0.0004$ (t-test).

same chance to interact with beads, more beads can be internalised. In this study, time-lapse image analyses clearly demonstrated that the migratory distance travelled by macrophages was not changed after activation with MAFs. 
The same time-lapse imaging revealed a significant increase in phagocytic activity after MAF activation (data not shown). According to these results, the first hypothesis was falsified.

The second hypothesis was then investigated with two experiments. First, the efficiency of engulfment was calculated as the ratio of internalised to attached beads. This was obtained by careful observation of time-lapse images. The ratio was significantly increased by serum MAF treatment. Second, the phagocytic activity was analyzed analysed in a continuously stirring condition, since macrophages can exert their phagocytic activity in suspension conditions (8). Additionally, in this experiment, the efficiency of engulfment was increased by serum MAF treatment, and this increase was higher than that in the former experiment. This might be due to the higher probability of macrophages interacting with beads with stirring. These two results consistently support the hypothesis that the activation of phagocytic activity with serum MAF is attributed to the augmentation of the efficiency of engulfment.

According to the time-lapse images, when the macrophage was activated with serum MAF, it started to engulf beads immediately after it attached to them. However, the macrophages that were not activated had a lag time between attachment to the beads and engulfment. During this lag time, the macrophages continued moving and appeared to be pushing the beads. Moreover, activated macrophages clearly showed active ruffling at the periphery of the cell. Since, both membrane ruffling and phagocytic cup formation are due to the active reorganization of actin filaments lining the plasma membrane $(9,10)$, the active ruffling and augmentation of efficiency of engulfment might be related.

In summary, our studies revealed that the phagocytic activation of macrophages with serum MAF was related to the efficiency of engulfment, but not to the migratory activity. Hence, our data suggest that the ruffling of cell membranes and lamellipodia are important for increase in the phagocytic activity of macrophages with the serum MAF. This study contributes to the understanding of activation of the immune system through the induction of the phagocytic activity of macrophages.

\section{Acknowledgements}

The Authors thank Dr. T. Inui (Inui Immunotherapy Clinic, Osaka, Japan) and Dr. Y. Uto (Tokushima University Graduate School, Tokushima, Japan) for providing serum MAF.

\section{References}

1 Zhang L and Wang CC: Inflammatory response of macrophages in infection. HBPD Int 13: 138-152, 2014.

2 Unanue ER: Antigen-presenting function of the macrophage. Ann Rev Immunol 2: 395-428, 1984.

3 Reeves AR, Spiller KL, Freytes DO, Vunjak-Novakovic G and Kaplan DL: Controlled release of cytokines using silkbiomaterials for macrophage polarization. Biomaterials 73: 272283, 2015.

4 Inoue T, Ishikawa M, Sumiya Y, Kohda H, Inui T, Kuchiike D, Kubo K, Uto Y and Nishikata T: Establishment of a macrophage activating factor (MAF) assay system using human-monocytic cell line, THP-1. Anticancer Res 35: 4441-4446, 2015.

5 Yamamoto $\mathrm{N}$ and Homma S: Vitamin D3 binding protein (group specific component) is precursor for the macrophage-activating signal factor from lysophosphotidylcholine-treated lymphocytes. Proc Natl Acad Sci USA 88: 8539-8543, 1991.

6 Inui T, Kuchiike D, Kubo K, Mette M, Uto Y, Hori H and Sakamoto $\mathrm{N}$ : Clinical experience of integrative cancer immunotherapy with GcMAF. Anticancer Res 33: 2917-2920, 2013.

7 Ishikawa M, Mashiba R, Kawakatsu K, Tran NK and Nishikata T: A high-throughput quantitative assay system for macrophage phagocytic activity. Macrophage 5: e1627, 2018.

8 Williams MR, Cauvi DM, Rivera I, Hawisher D and De Maio A: Changes in macrophage function modulated by the lipid environment. Innate Immun 22: 141-151, 2016.

9 Ohsawa K, Imai Y, Kanazawa H, Sasaki Y and Kohsaka S: Involvement of Iba1 in membrane ruffling and phagocytosis of macrophages/microglia. J Cell Sci 113: 3073-3084, 2000.

10 Swanson JA: Shaping cups into phagosomes and macropinosomes. Nat Rev Mol Cell Biol 9: 639-649, 2008.
Received April 11, 2018

Revised May 15, 2018

Accepted May 16, 2018 